\section{Irradiation of food of animal origin: analytical checks to verify compliance with EU legislation in Lazio region}

\author{
Selene Marozzi, ${ }^{1}$ Roberto Condoleo, ${ }^{1}$ \\ Stefano Saccares, ${ }^{1}$ \\ Maria Concetta Campagna, ${ }^{2}$ \\ Michele Mangiacotti, ${ }^{3}$ \\ Eugenio Chiaravalle ${ }^{3}$ \\ ${ }^{1}$ Centro Studi per la Sicurezza \\ Alimentare, Istituto Zooprofilattico \\ Sperimentale delle Regioni Lazio e \\ Toscana, Roma; 'Direzione operativa \\ Produzioni Zootecniche, Istituto \\ Zooprofilattico Sperimentale delle \\ Regioni Lazio e Toscana, Roma; ${ }^{3}$ Centro di \\ Referenza Nazionale per la Ricerca della \\ Radioattività nel Settore Zootecnico \\ Veterinario - Struttura Complessa \\ Chimica, Istituto Zooprofilattico \\ Sperimentale della Puglia e della \\ Basilicata, Foggia, Italy
}

\section{Abstract}

Irradiation is a method of food preservation regulated in the European Union (EU) by Directives 1999/2/EC and 1999/3/EC, and adopted in Italy with Legislative Decree 94, $30 / 01 / 2001$. The aim of this paper is to present the results of control in Lazio Region done to verify compliance with the mandatory legislation. For this purpose, 36 samples of food of animal origin marketed in Lazio were collected and analysed. Of these, $11.2 \%$ was irradiated and the treatment was not provided on the label.

\section{Introduzione}

Si definiscono ionizzanti le onde elettromagnetiche di bassa lunghezza ed elevata frequenza $(f>3000 \mathrm{THz},<0,1 \mathrm{~m})$ che, interagendo con la materia, determinano la ionizzazione degli atomi esposti alla loro azione per rimozione di elettroni dagli orbitali esterni (Bibek e Bhunia, 2008).

Le radiazioni ionizzanti vengono utilizzate nell'industria alimentare per diversi scopi, tra i quali: prevenire la germinazione e germogliazione di patate, cipolle ed aglio, ritardare la maturazione e l'invecchiamento di frutta e verdura, aumentare la shelf-life degli alimenti, e migliorarne la qualità igienico sanitaria (Stefanova et al., 2010; Farkas, 1998).

L'irraggiamento degli alimenti è sottoposto nell'Unione Europea (UE) a specifiche condi- zioni di applicabilità sancite dalla Direttive 1999/2/CE e 1999/3/CE (Commissione Europea, 1999a; Commissione Europea, 1999b), recepite in Italia con il DL 94 del 30/01/2001 (Repubblica Italiana, 2001). Secondo le disposizioni legislative cogenti, gli alimenti 0 ingredienti irradiati, destinati al consumatore finale 0 alla collettività, devono riportare in etichetta la dicitura irradiato o trattato con radiazioni ionizzanti. Nella nostra nazione è attualmente consentito l'irraggiamento di: i) erbe aromatiche essiccate, spezie e condimenti vegetali (Commissione Europea, 1999a; Commissione Europea, 1999b; Repubblica Italiana, 2001); e ii) agli, patate, cipolle (DM 30/08/1973 articolo 2, comma 2; Repubblica Italiana, 1973). Le normative vigenti nella UE impongono ad ogni nazione l'esecuzione di controlli sugli alimenti nella fase di commercializzazione e sugli stabilimenti che effettuano il trattamento. Nella Regione Lazio, a partire dal 2011, le attività di controllo in fase di commercializzazione (in precedenza su base volontaria) sono state pianificate per i prodotti di origine animale. Scopo del presente lavoro è illustrare i risultati dei controlli effettuati.

\section{Materiali e Metodi}

Lo studio di tipo osservazionale con campionamento non probabilistico per quote (convenience sampling), è stato condotto secondo gli assunti seguenti: i) popolazione target: esercizi di vendita al dettaglio; ii) unità epidemiologiche: alimenti di origine animale a maggior rischio di non conformità (DG SANCO, 2012) ovvero cosce di rana, carcasse di pollo, teleostei, gamberetti e molluschi eduli lamellibranchi, non etichettati con l'indicazione dell'avvenuto trattamento, provenienti da Paesi terzi o da altri Stati membri CE nei quali insistono impianti riconosciuti per l'irraggiamento dei prodotti di origine animale; iii) campioni minimi stabiliti: $\mathrm{n}=44$ stratificati per singole ASL regionali. Le attività di campionamento sono state effettuate secondo le disposizioni della Legge 30 aprile 1962, n. 283 (Repubblica Italiana, 1962), del DPR 327 del 26/03/1980 (Repubblica Italiana, 1980) e del DL 3 marzo 1993, n. 123 e successive modifiche (Repubblica Italiana, 1993). I campioni selezionati in base al criterio di casualità sono stati inviati ai laboratori a temperatura di refrigerazione 0 congelati in apposite confezioni al fine di evitare esposizioni a fonti luminose o di calore che potessero influenzare il risultato delle successive analisi. Le indagini di laboratorio sono state condotte con metodiche di tipo biologico 0 fisico stadardizzate dal Comitato Europeo di Normazione (CEN)come di seguito specificato: i) carne di pollo contenente ossa: UNI EN 13784:2002 (UNI, 2002b), ovvero screening biologico (DNA comet assay) basato sulla frammentazione del
Correspondence: Selene Marozzi, Centro Studi per la Sicurezza Alimentare, Istituto Zooprofilattico Sperimentale delle Regioni Lazio e Toscana, via Appia Nuova 1411, 00178 Roma, Italy.

Tel. +39.06 .7099360 - Fax: +39.06 .7099312 .

E-mail: selene.marozzi@izslt.it

Key words: Food irradiation, Products of animal origin, Non-compliant samples.

Conflict of interests: the authors declare no potential conflict of interests.

Received for publication: 15 January 2013.

Revision received: 25 March 2013.

Accepted for publication: 25 March 2013.

This work is licensed under a Creative Commons Attribution 3.0 License (by-nc 3.0).

(C) Copyright S. Marozzi et al., 2013

Licensee PAGEPress, Italy

Italian Journal of Food Safety 2013; 2:e17

doi:10.4081/ijfs.2013.e17

DNA indotta da radiazioni ionizzanti, evidenziata con l'impiego di elettroforesi su gel; ii) carne di pollo, cosce di rana e teleostei contenenti ossa: UNI EN 1786:1997 (UNI, 1997), ovvero metodo fisico di conferma basato sulla misurazione, mediante risonanza di spin elettronico [electron spin resonance (ESR)], dei radicali liberi; iii) crostacei e molluschi: UNI EN 13751:2009 (UNI, 2009) e UNI EN 1788:2002 (UNI, 2002a), rispettivamente metodo fisico di screening e di conferma, basati sulla misura della luce di luminescenza emessa per stimolazione ottica [photo-stimulated luminescence (PSL)] 0 termica (termoluminescenza) dei minerali (silicati o carbonati) presenti negli alimenti come costituenti o contaminanti.

\section{Risultati}

Sono stati sottoposti ad analisi $\mathrm{n}=36$ campioni corrispondenti all' $81,8 \%$ del totale dei campioni minimi stabiliti nel piano $(n=44)$. Di questi, l'11,1\% $(n=4)$ sono risultati non conformi, ovvero sottoposti a trattamento con radiazioni ionizzanti non dichiarato in etichetta. Tutti gli alimenti irraggiati appartenevano alla matrice cosce di rana (Tabella 1).

\section{Discussione}

A differenza di quanto accade in altri Stati membri (per esempio Francia ed Olanda) ed in alcuni Paesi terzi (per esempio USA), nella nostra nazione non è concesso l'irraggiamento 
Tabella 1. Regione Lazio: piano di controllo alimenti di origine animale irraggiati: campioni analizzati ed esiti analitici.

\begin{tabular}{|c|c|c|c|c|c|c|c|}
\hline \multirow[t]{2}{*}{$\begin{array}{l}\text { Tipologia alimenti } \\
\text { analizzati }\end{array}$} & \multirow[t]{2}{*}{$\begin{array}{l}\text { Campioni } \\
\text { effettuati }\end{array}$} & \multirow[t]{2}{*}{$\begin{array}{l}\text { Campioni } \\
\text { attesi }\end{array}$} & \multirow[t]{2}{*}{$\begin{array}{l}\text { Conformi } \\
\text { (\% sul totale) }\end{array}$} & \multirow[t]{2}{*}{ Dubbi } & \multirow[t]{2}{*}{$\begin{array}{l}\text { Non conformi } \\
\text { (\% sul totale) }\end{array}$} & \multicolumn{2}{|c|}{$\begin{array}{c}\text { Metodo CEN } \\
\text { utilizzato }\end{array}$} \\
\hline & & & & & & Screening & Conferma \\
\hline Cosce di rana & 9 & 8 & $5(55,6 \%)$ & 0 & $4(44,4 \%)$ & - & $\begin{array}{l}\text { UNI EN } \\
1786: 1997 \\
\text { (UNI, 1997) }\end{array}$ \\
\hline $\begin{array}{l}\text { Carne di pollo } \\
\text { contenente osso }\end{array}$ & 8 & 12 & $8(100 \%)$ & 0 & 0 & $\begin{array}{l}\text { UNI EN } \\
13784: 2002 \\
\text { (UNI, 2002b) }\end{array}$ & $\begin{array}{l}\text { UNI EN } \\
1786: 1997 \\
\text { (UNI, 1997) }\end{array}$ \\
\hline Teleostei contenenti osso & 9 & 12 & $9(100 \%)$ & 0 & 0 & - & $\begin{array}{l}\text { UNI EN } \\
1786: 1997 \\
\text { (UNI, 1997) }\end{array}$ \\
\hline $\begin{array}{l}\text { Gamberetti e molluschi } \\
\text { lamellibranchi }\end{array}$ & 10 & 12 & $10(100 \%)$ & 0 & 0 & $\begin{array}{l}\text { UNI EN } \\
13751: 2009 \\
(\text { UNI, 2009) }\end{array}$ & $\begin{array}{l}\text { UNI EN } \\
1788: 2002 \\
\text { (UNI, 2002a) }\end{array}$ \\
\hline Totale & 36 & 44 & $32(88,8 \%)$ & 0 & $4(11,2 \%)$ & - & \\
\hline
\end{tabular}

CEN, Comitato Europeo di Normazione.

di prodotti di origine animale. Ciononostante, è possibile che in Italia siano commercializzati alimenti di origine animale irradiati in altre nazioni. Infatti, indipendentemente da tale divieto, l'apertura dei mercati europei consente l'immissione sul mercato interno di prodotti trattati negli Stati membri comunitari.

In Italia i primi controlli sul rispetto della normativa cogente sono stati effettuati nel 2005. Nel 2010 è stato riscontrato il primo prodotto di origine animale irregolare, il quale, in linea con quanto rilevato nella Regione Lazio nel 2011, era un campione di cosce di rana (DG SANC0, 2012). Sebbene l'irraggiamento in sé possa non comportare un rischio per la salute del consumatore, è tuttavia obbligatorio secondo le attuali disposizioni normative, indicare l'avvenuto trattamento in etichetta (Commissione Europea, 1999a). In merito, invece, al potenziale rischio tossicologico correlato a trattamenti con dosi globali medie superiori a $10 \mathrm{kGy}$ (Stefanova et al., 2010), va specificato che i metodi analitici attuali non sono in grado di determinare la dose utilizzata per l'irraggiamento e di conseguenza risultano inefficaci nel comprovare la sicurezza tossicologica dell'alimento.

\section{Conclusioni}

In conclusione, sulla base dei risultati riferiti nei report comunitari, nazionali e del presente lavoro, appare evidente implementare i controlli su alcune tipologie di prodotto. A tal proposito, l'Area Sanità Veterinaria della Regione Lazio ha approvato un ulteriore e più articolato programma di campionamento per l'anno 2012.

\section{Bibliografia}

Bibek R, Bhunia A, 2008. Fundamental food microbiology. 4th ed. CRC Press, Boca Raton, FL, USA.

Commissione Europea, 1999a. Direttiva del Parlamento europeo e del Consiglio del 22 febbraio 1999 relativa al ravvicinamento delle legislazioni degli Stati membri concernenti gli alimenti e i loro ingredienti trattati con radiazioni ionizzanti, 1999/2/CE. In: Gazzetta Ufficiale, L 066, 13/03/1999, pp 16-23.

Commissione Europea, 1999b. Direttiva del Parlamento europeo e del Consiglio del 22 febbraio 1999 che stabilisce un elenco comunitario di alimenti e loro ingredienti trattati con radiazioni ionizzanti, 1999/3/CE. In: Gazzetta Ufficiale, L 66/24, 13/03/1999.

DG SANCO, 2012. Food irradiation. DirectorateGeneral for Health and Consumers, European Commission ed., Brussels, Belgium. Disponibile al sito: http//ec.europa.eu/food/food/biosafety/irradiation/index_en.htm

Farkas J, 1998. Irradiation as a method for decontaminating food: a review. Intl J Food Microbiol 44:189-204.

Repubblica Italiana, 1962. Modifica degli articoli $242,243,247,250$ e 262 del testo unico delle leggi sanitarie, approvato con regio decreto 27 luglio 1934, n. 1265: Disciplina igienica della produzione e della vendita delle sostanze alimentari e delle bevande, L139, 04/06/1962.

Repubblica Italiana, 1973. Autorizzazione al trattamento mediante radiazioni gamma, a scopo antigermogliativo, di patate, cipolle ed agli, DM 30/08/1973. In: Gazzetta Ufficiale no. $254,01 / 10 / 1973$.

Repubblica Italiana, 1980. Regolamento di ese- cuzione della L. 30 aprile 1962, n.283, e successive modificazioni, in materia di disciplina igienica della produzione e della vendita delle sostanze alimentari e delle bevande, 327/1980. In: Gazzetta Ufficiale no. 193, 16/07/1980.

Repubblica Italiana, 1993. Attuazione della direttiva 89/397/CEE relativa al controllo ufficiale dei prodotti alimentari, DL 123/1993. In: Gazzetta Ufficiale no. 97, 27/04/1993.

Repubblica Italiana, 2001. Attuazione delle direttive 1999/2/CE e 1993/3/CE concernenti gli alimenti e i loro ingredienti trattati con radiazioni ionizzanti, DL 94/2001 del 30 gennaio 2001. In: Gazzetta Ufficiale no. 79, 04/04/2001.

Stefanova R, Vasilev VN, Sapassov SL, 2010. Irradiation of food, current legislation frame work and detection of irradiated foods. Food Anal Method 3:225-52.

UNI, 1997. Norma UNI EN 1786:1997. Prodotti alimentari. Ricerca di alimenti irraggiati contenenti ossa. Metodo per spettroscopia di risonanza elettronica di spin (ESR). Ente Nazionale Italiano di Unificazione ed., Milano, Italia.

UNI, 2002a. Norma UNI EN 1788:2002. Prodotti alimentari: ricerca per termoluminescenza di alimenti irraggiati dai quali possono essere isolati i minerali silicati. Ente Nazionale Italiano di Unificazione ed., Milano, Italia.

UNI, 2002b. Norma UNI EN 13784:2002. Prodotti alimentari: saggio comet Dna per la ricerca di prodotti alimentari irraggiati - metodo per screening. Ente Nazionale Italiano di Unificazione ed., Milano, Italia.

UNI, 2009. Norma UNI EN 13751:2009. Prodotti alimentari: ricerca di alimenti irraggiati con l'utilizzo di luminescenza fotostimolata. Ente Nazionale Italiano di Unificazione ed., Milano, Italia. 